\title{
Days alive and out of hospital within 90 days after fast-track total hip and knee arthroplasty
}

\author{
C. C. Jørgensen, H. Kehlet, The Lundbeck Foundation Centre for Fast-track Hip and Knee Replacement \\ Collaborative Group
}

Section for Surgical Pathophysiology and the Lundbeck Foundation Centre for Fast-track Hip and Knee Replacement Collaboration,

Rigshospitalet, Copenhagen University - Copenhagen (Denmark)

Background and Goal of study: Days alive and out of hospital (DAOH) has been proposed as an alternative pragmatic outcome measure for medical ${ }^{1}$, and perioperative outcome studies ${ }^{2}$. However, the lack of procedure specific data and standardized perioperative care may hinder interpretation. The goal of this study was to investigate DAOH within 90-days after fast-track total hip (THA) and knee arthroplasty (TKA)

Methods: Cohort study in 8 centers from 2012-2015 with prospective data on preoperative characteristics and complete follow-up on length of hospital stay (LOS) and 90-days readmissions/mortality using the Danish National Patient Registry. LOS was calculated as postoperative nights in hospital. Readmissions were included if related to the surgical procedure and counted as days in hospital, excluding emergency room/outpatient visits. High-risk patients were defined according to a previously published definition based on preoperative characteristics. ${ }^{3}$

Table 1. Fractions of patients according to $\mathrm{DAOH}$

\begin{tabular}{lllll}
\hline & DAOH 0-10 & DAOH $0-45$ & DAOH 0-60 & DAOH 0-80 \\
all patients $(\mathrm{n}: 16137)$ & $0.1 \%$ & $0.4 \%$ & $0.7 \%$ & $3.9 \%$ \\
high-risk patients $^{1}(\mathrm{n}: 2889)$ & $0.3 \%^{*}$ & $1.0 \%^{* *}$ & $1.5 \% \%^{* *}$ & $9.2 \%{ }^{* *}$ \\
low-risk patients $(\mathrm{n}: 12633)$ & $0.1 \%$ & $0.3 \%$ & $0.5 \%$ & $2.7 \%$ \\
\hline
\end{tabular}

$1 \geq 2$ of the following preoperative characteristics: age $\geq 80$ years, use of walking aids, anemia, pulmonary disease, psychiatric disorder, anticoagulant therapy ${ }^{3}$. $\mathrm{DAOH}$ : days alive and out of hospital. ${ }^{*} \mathrm{p}=0.001{ }^{* *} \mathrm{p}<0.001 \mathrm{vs}$. low-risk patients.

Results: Of 16137 procedures, 2889 (19\%, range 12-23\% between departments) were in high-risk and 12633 $(81 \%)$ in low-risk patients, (615 patients excluded due to missing data.) Median LOS was 2 days (IQR 2-3), 90 -days readmissions were $8.2 \%$ and all-cause mortality was $0.3 \%$. The median DAOH was 88 days (IQR $87-$ 88 ) and $>96 \%$ of patients had DAOH between 81 and 90 days.

The fraction with DAOH $81-90$ was higher $(90.8 \%$ vs. $97.3 \%)$ in high vs. low-risk patients $(p<0.001)$, as was the fraction with $\mathrm{DAOH} \leq 10, \leq 45, \leq 60$ and $\leq 80$. (table 1 and fig. 1 )

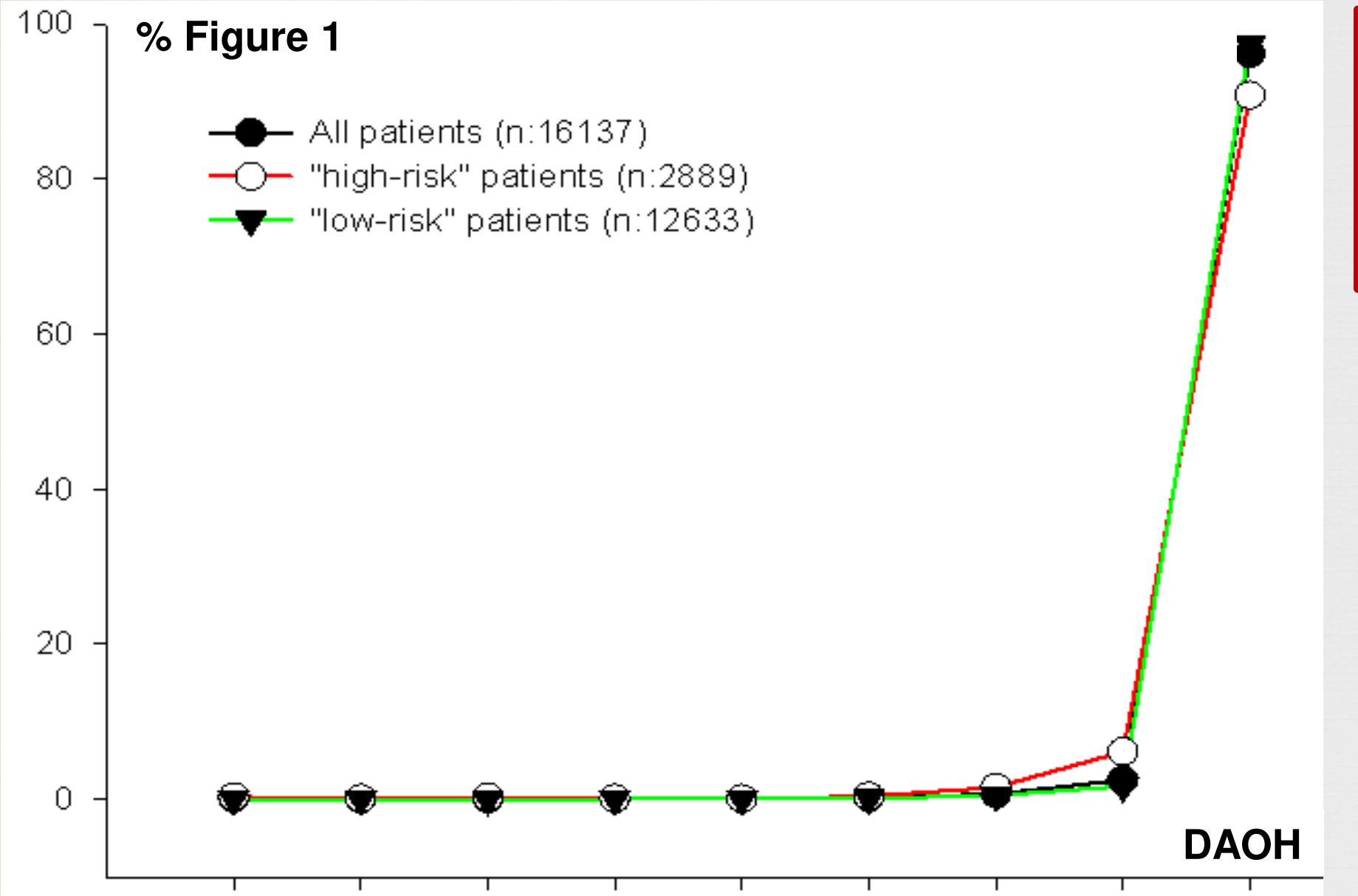

$0-10 \quad 11-20 \quad 21-30 \quad 31-40 \quad 41-50 \quad 51-60 \quad 61-70 \quad 71-80 \quad 81-90$
CONCLUSION: High-risk patients had fewer DAOH. However, the clinical relevance of 90 -days $\mathrm{DAOH}$ as a pragmatic outcome measure may be limited in fasttrack THA and TKA.

Discussion: High-risk patients had fewer DAOH. However, in contrast to a study in mixed procedures measuring 30 days $\mathrm{DAOH}$ [2], the clinical relevance of 90 -days $\mathrm{DAOH}$ in our study was limited considering the rarity of $\mathrm{DAOH}<81$, also in high-risk patients. Furthermore, DAOH does not provide information on postoperative morbidity, prohibiting analysis on what went wrong. 